Article

\title{
Use of Anion Exchange Resins for One-Step Processing of Algae from Harvest to Biofuel
}

\author{
Jessica Jones ${ }^{1}$, Cheng-Han Lee ${ }^{2}$, James Wang ${ }^{2}$ and Martin Poenie ${ }^{2, *}$
}

1 Department of Biomedical Engineering, University of Texas at Austin, Austin, TX 78712, USA; E-Mail: jessica.jones@utexas.edu

2 Section of Molecular, Cell and Developmental Biology, University of Texas at Austin, Austin, TX 78712, USA; E-Mails: hanleex@utexas.edu (C.-H.L); james.wang@utsouthwestern.edu (J.W.)

* Author to whom correspondence should be addressed; E-Mail: poenie@mail.utexas.edu; Tel.: +1-512-471-5598; Fax: +1-512-471-3878.

Received: 15 March 2012; in revised form: 5 June 2012 / Accepted: 13 July 2012 /

Published: 24 July 2012

\begin{abstract}
Some microalgae are particularly attractive as a renewable feedstock for biodiesel production due to their rapid growth, high content of triacylglycerols, and ability to be grown on non-arable land. Unfortunately, obtaining oil from algae is currently cost prohibitive in part due to the need to pump and process large volumes of dilute algal suspensions. In an effort to circumvent this problem, we have explored the use of anion exchange resins for simplifying the processing of algae to biofuel. Anion exchange resins can bind and accumulate the algal cells out of suspension to form a dewatered concentrate. Treatment of the resin-bound algae with sulfuric acid/methanol elutes the algae and regenerates the resin while converting algal lipids to biodiesel. Hydrophobic polymers can remove biodiesel from the sulfuric acid/methanol, allowing the transesterification reagent to be reused. We show that in situ transesterification of algal lipids can efficiently convert algal lipids to fatty acid methyl esters while allowing the resin and transesterification reagent to be recycled numerous times without loss of effectiveness.
\end{abstract}

Keywords: algae; biodiesel; biofuels; concentration; dewatering; direct transesterification; harvest 


\section{Introduction}

Some strains of algae show promise as a sustainable source of biofuel due to their rapid growth, ability to grow on non-arable land, and high triacylglycerol (TAG) content. TAGs can be easily converted to biodiesel, which is compatible with current fuel infrastructure [1]. Biodiesel has a higher energy density than ethanol, yet it is relatively non-toxic, biodegradable, and produces lower exhaust emissions than petroleum-based fuels, making it is one of the most attractive forms of alternative energy [2,3]. Additionally, when derived from plant sources such as algae, biodiesel has the potential of a near-neutral carbon footprint [4], though considerable work must be done in order to realize this goal in an economically and environmentally-feasible manner [5].

While algae are promising, there are technical challenges that currently make it prohibitively expensive as a source of fuel. Algae grow at dilute concentrations, generally less than $1 \mathrm{~g} / \mathrm{L}$ [6], so it must be concentrated before it can be processed. Most concentration processes require pumping the dilute algal suspension and may involve other energy intensive steps such as centrifugation [7], compressors for dissolved air flotation, or else treatment of large volumes of water with chemicals (flocculation). Centrifugation, for example, could account for $30 \%$ of processing costs [8].

Once algae have been harvested, there are additional processing steps that often include lysis and drying of the algae followed by extraction with organic solvents to obtain the neutral lipids, primarily TAGs, that can then be converted to biodiesel [3]. Drying can be expensive but it facilitates the interaction between solvent and algae to improve extraction efficiency. However, the extraction solvent must be removed and recovered prior to conversion of lipids to biodiesel and there are attending questions about pollution of the air and contamination of the biomass with solvents. This is also considered a large part of the cost in processing algae [5].

Once the lipids have been isolated, they can be converted to biodiesel by either acid- or base-catalyzed transesterification, typically with methanol, to yield fatty acid methyl esters (FAMEs) and glycerol [9]. The transesterification reaction is sensitive to water [10-14] but this is not normally a problem since oils, such as would be obtained by hexane extraction, normally contain little water. When the entire processes is analyzed for cost and energy expenditure, some current projections suggest that the energy spent in the cultivation, harvest, and extraction of oil from algae could be greater than that gained from the product [5] along with the possibility of significant environmental impact [15]. It is generally agreed that for algae to be economically feasible, improvements in technology will be needed.

Efforts to reduce cost and simplify processing of algal biomass to biofuel have led to studies of direct conversion of dry or even wet algal biomass to biodiesel. Previous studies have shown that algal lipids can be transesterified in situ by adding reagents to a dried sample of algae [11,16-19]. One study examined a two-step procedure where the acyl groups of component lipids were hydrolyzed with base and then re-esterified in excess sulfuric acid/methanol [20]. This procedure gave greater amounts FAME than obtained by lipid extraction followed by transesterification. Another study showed that direct acid-catalyzed transesterification of wet biomass can produce FAME yields similar to that of dried biomass, although FAME compositions differed [11].

In this study we show that anion exchange resins such as Amberlite can concentrate and dewater algae (i.e., harvest algae) and then be eluted with 5\% sulfuric acid/methanol reagent. The eluted algae 
appear to dissolve in the sulfuric acid reagent and esterified fatty acids are converted to FAMEs (biodiesel). This one step harvesting and transesterification process can potentially eliminate many of the costly steps of processing algae to biofuel.

\section{Results and Discussion}

\subsection{Algae Are Harvested and Concentrated onto Amberlite Anion Exchange Resin}

Amberlite CG-400, a divinylbenzene-based resin containing quaternary ammonium groups (3.8 $\mathrm{mmol} / \mathrm{g}$ [21]), has been previously shown to bind and concentrate two different species of green algae for removal from the water supply [22]. Here, we used Amberlite to concentrate and dewater biomass from two potentially high oil-producing green algal species, Neochloris oleoabundans [23] and KAS 603 [24]. For routine comparisons, algal suspension was passed through the column until saturation was achieved. Algae appeared to bind on contact, initially accumulating as a band at the top of the resin bed. As more algae were added, the upper green layer progressively expanded down to the bottom of the resin bed. Prior to the resin bed becoming solid green, the flow-through was clear and colorless. Once the resin bed became solid green, color quickly began to appear in the effluent and this was taken as the saturation point.

To determine the binding capacity of the resin for Neochloris and KAS 603, the $\mathrm{OD}_{680}$ of the algal suspension was measured before and after passing the algae through an Amberlite column. The concentration of algae in the initial suspension and the in flow-through were determined from algal $\mathrm{OD}_{680}$ absorbance versus dry cell weight (DCW) calibration curves generated for both types of algae (Supplementary Figure S1). The flow-through included the solution from the algae algal suspension that went through the resin and the subsequent wash to remove unbound algae. The total amount of algae bound to the resin was then determined by subtracting the amount of algae in the flow-through from the total that was added to the column.

For all the experiments algal binding to the resin was tested at $\mathrm{pH} \mathrm{7,} \mathrm{although} \mathrm{there} \mathrm{was} \mathrm{no}$ significant difference in binding over the $\mathrm{pH}$ range of 6-9 (Supplementary Figure S2). Since for Amberlite, algal binding decreases with increasing ionic strength, binding studies for both algae were tested under freshwater conditions (salinity $5 \mathrm{psu}$ ). The algal suspensions were used at $0.4 \mathrm{~g} / \mathrm{L}$, the value that we typically obtain in our simple airlift photobioreactors. When more dilute algal suspensions were tested, saturation of the resin was still achieved but, as would be expected, the volume of algal suspension and time required to achieve saturation increased. Since algae can be entrained in the resin beads, our protocol included a washing step between binding and elution to ensure only algae that were actually bound to the resin were counted.

Figure 1(a) gives a schematic for resin-binding and elution experiments shown in Figures 1-3. Algal binding capacity was determined for each cycle of algal binding. Reusability of the resin was assessed in terms of loss of binding capacity over multiple cycles of algal binding and elution. These experiments were carried out either using only fresh sulfuric acid/methanol reagent to elute the algae (Series A, "fresh") or using sulfuric acid/methanol that was recycled after the first use (Series B, "used"). 
Although the flow through approach in Figure 1(a) gave repeatable values for saturation and was used for all experiments except that shown in Figure 4, we later found that longer contact times, which involved stirring excess algae with resin for up to $15 \mathrm{~min}$, gave somewhat larger binding capacities than those obtained using the flow through method. However, while the binding capacity numbers would be improved with longer contact times, the patterns remain the same.

Figure 1. Algal binding capacity of Amberlite and resin reuse. (a) Experiment series for determining binding capacity and reusability of resin and transesterification reagent. (b) Binding capacities of Amberlite (mg algal dry weight / gram resin) were determined for Neochloris and KAS 603. (c) Neochloris or (d) KAS 603 were loaded onto resin columns and eluted with $100 \mathrm{~mL}$ of $5 \%$ sulfuric acid/methanol reagent. After washing the column with distilled water, the operation was repeated for three more cycles, eluting with either fresh methanol sulfuric acid reagent (fresh) or reusing the previously used reagent (used). The binding capacity of the resin was determined for each cycle of algal loading onto the resin.

a

Series A

Loading of Resin

Measure OD of algal suspension Add algal suspension to column of resin

Measure OD of flow-through

Calculate binding capacity

Elution of Resin
Add $100 \mathrm{~mL}$ fresh $\mathrm{H}_{2} \mathrm{SO}_{4} / \mathrm{MeOH}$ to
elute algae
Let eluate stand $12 \mathrm{~h}$ until
transesterification is complete
Extract eluate with hexane
Measure $\mathrm{FAME}$ content of extract
Discard eluate
Wash column with $\mathrm{H}_{2} \mathrm{O}$
Reuse resin

Series B

Loading of Resin

Measure OD of algal suspension Add algal suspension to column of resin

Measure OD of flow-through Calculate binding capacity

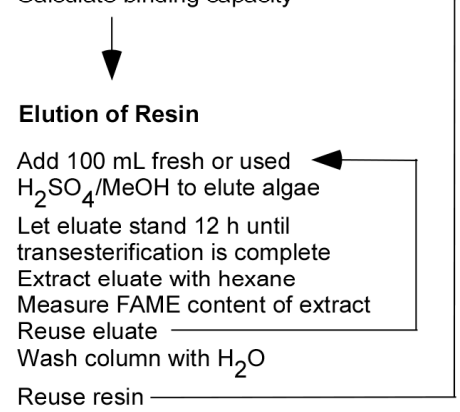

b

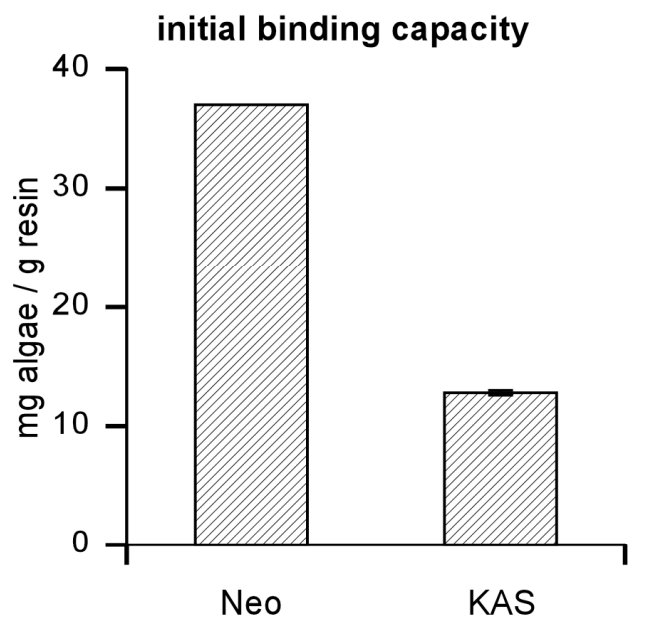

C

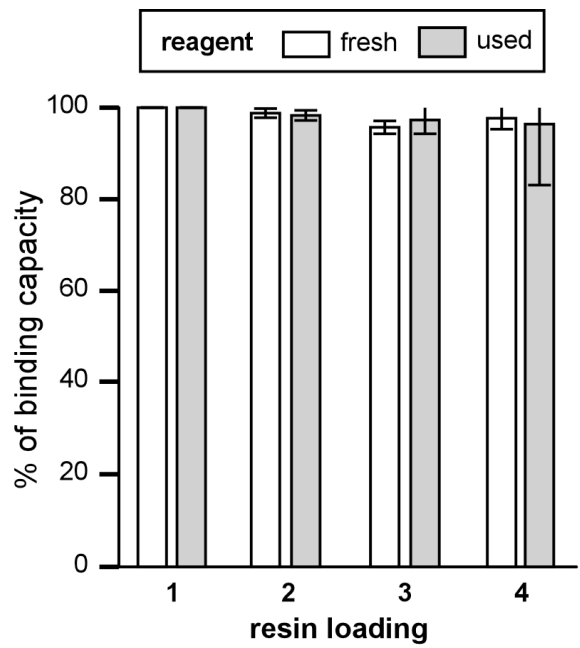

d

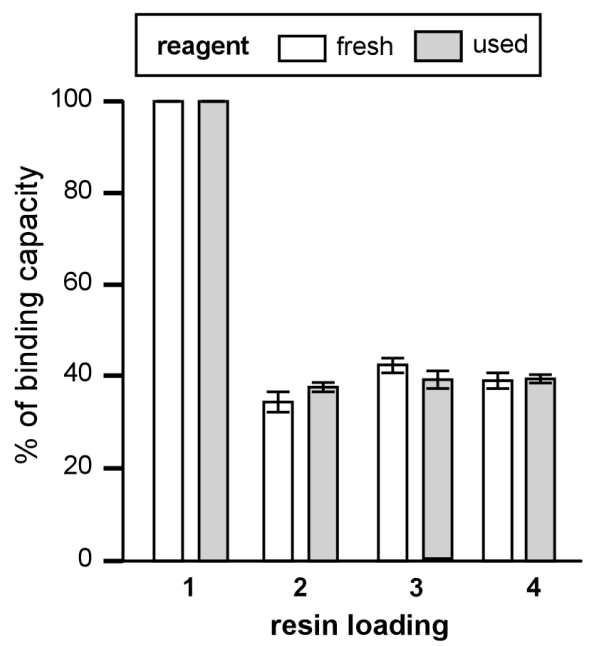


Figure 2. FAME yield and characterization by resin-bound transesterification. (a) FAMEs from normal or stressed (nitrogen-starved) Neochloris and KAS 603 were generated either by elution of resin-bound algae with $5 \%$ sulfuric acid/methanol reagent or by subjecting dry algal pellets subjected to sequential base hydrolysis followed by treatment with sulfuric acid in methanol to esterify free fatty acids. The FAME yields were expressed as percent of dry cell weight. The weight of a crude lipid extract from parallel batches of algae and the TAG content those extracts (determined by HPLC) are given for comparison. The FAME prepared from resin-bound algae were analyzed by HPLC/MS to determine the abundance of (b) saturated C16:0, C18:0, and $\mathrm{C} 20: 0$ and (c) unsaturated $\mathrm{C} 18: 3$ and $\mathrm{C} 21: 4$ acyl constituents relative to total FAME derived.

a

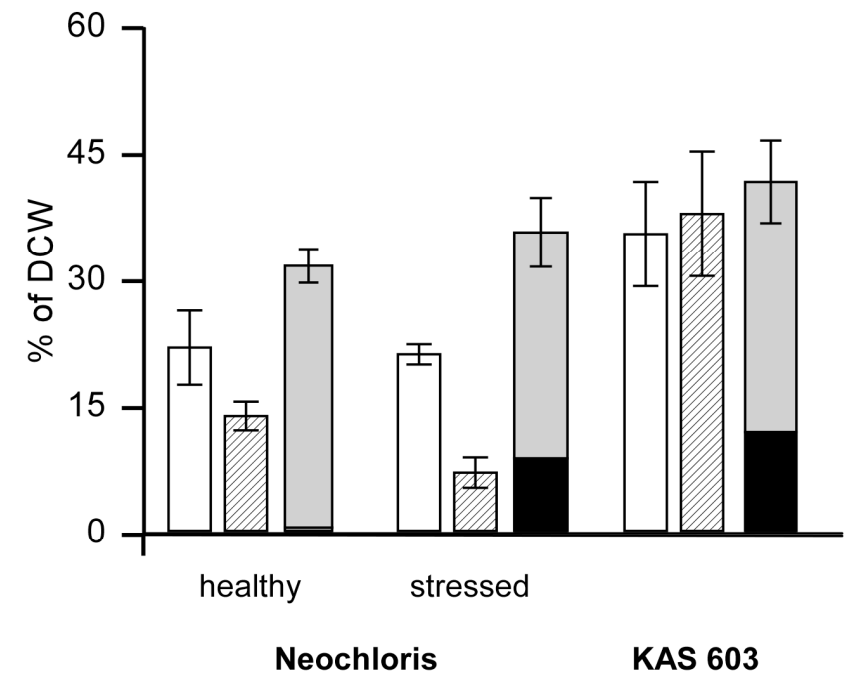

\section{FAME yield}
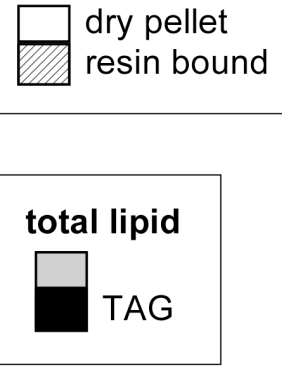
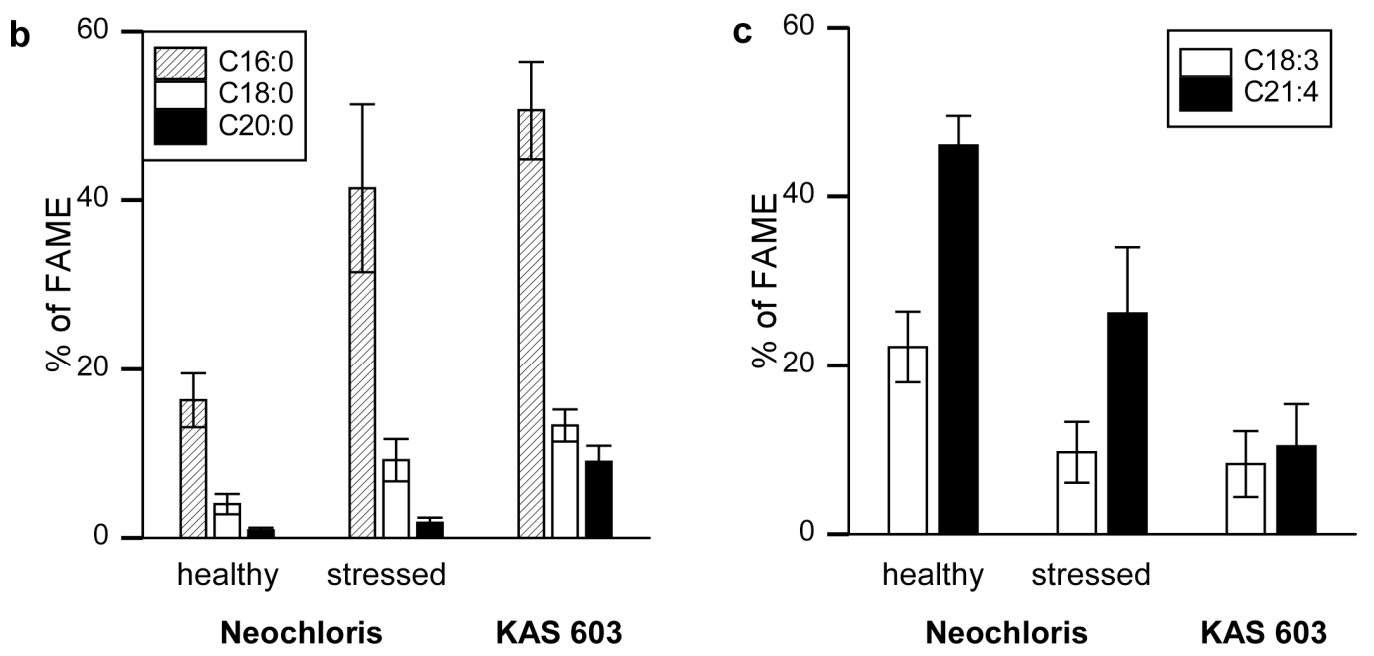
Figure 3. Efficiency of FAME production using recycled reagent, and FAME removal. (a) Algal were loaded onto Amberlite and eluted with 5\% sulfuric acid/methanol four times in succession using either fresh reagent or reusing the old reagent after the initial elution. Twelve $\mathrm{h}$ after each elution the FAME was extracted with hexane, solvent was removed and the amount of FAME determined as a percentage of algal dry cell weight; (b) The sulfuric acid/methanol reagent containing algal FAME was either extracted with hexane or, for comparison, passed over a hydrophobic resin column composed of $80 \%$ EGDMA and 20\% HMA; (c) FAME relative to total dry algal weight obtained by hexane extraction or after eluting FAME bound to the hydrophobic column.

a
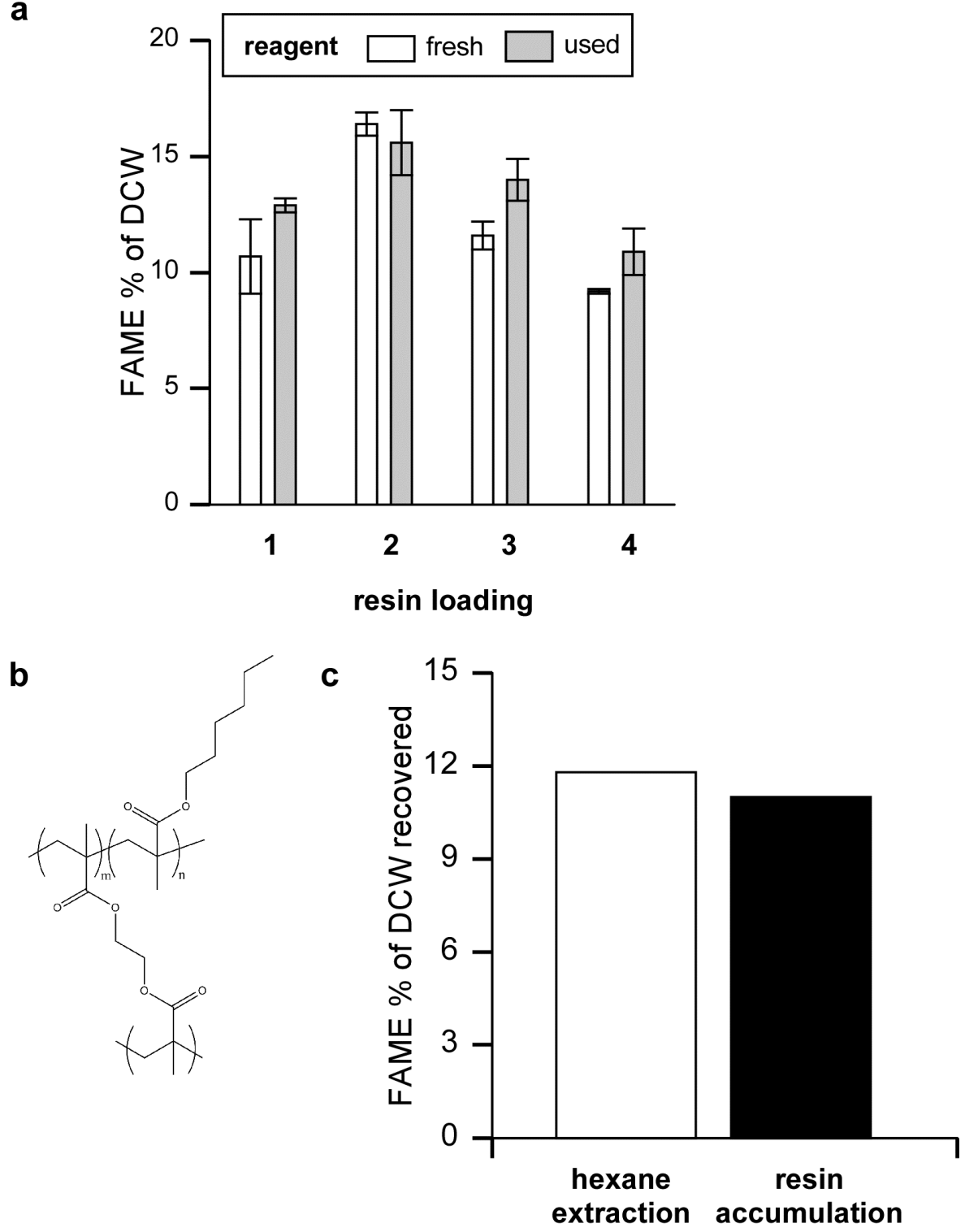

recovery method 
Figure 4. Functionalized resins for improved binding of KAS603. The relative binding capacity of Amberlite for KAS 603 is compared to anion exchange resins composed of either (a) EGDMA-IM-DEG (60:30:10) or (b) DVB-DMA (60:40); (c) The results show that the binding capacity of Amberlite is low compared to the other resins; (d) In addition to binding more algae, subsequent elution of either Amberlite or DVB-DMA shows that greater amounts of FAME are produced from the DVB:DMA resin.

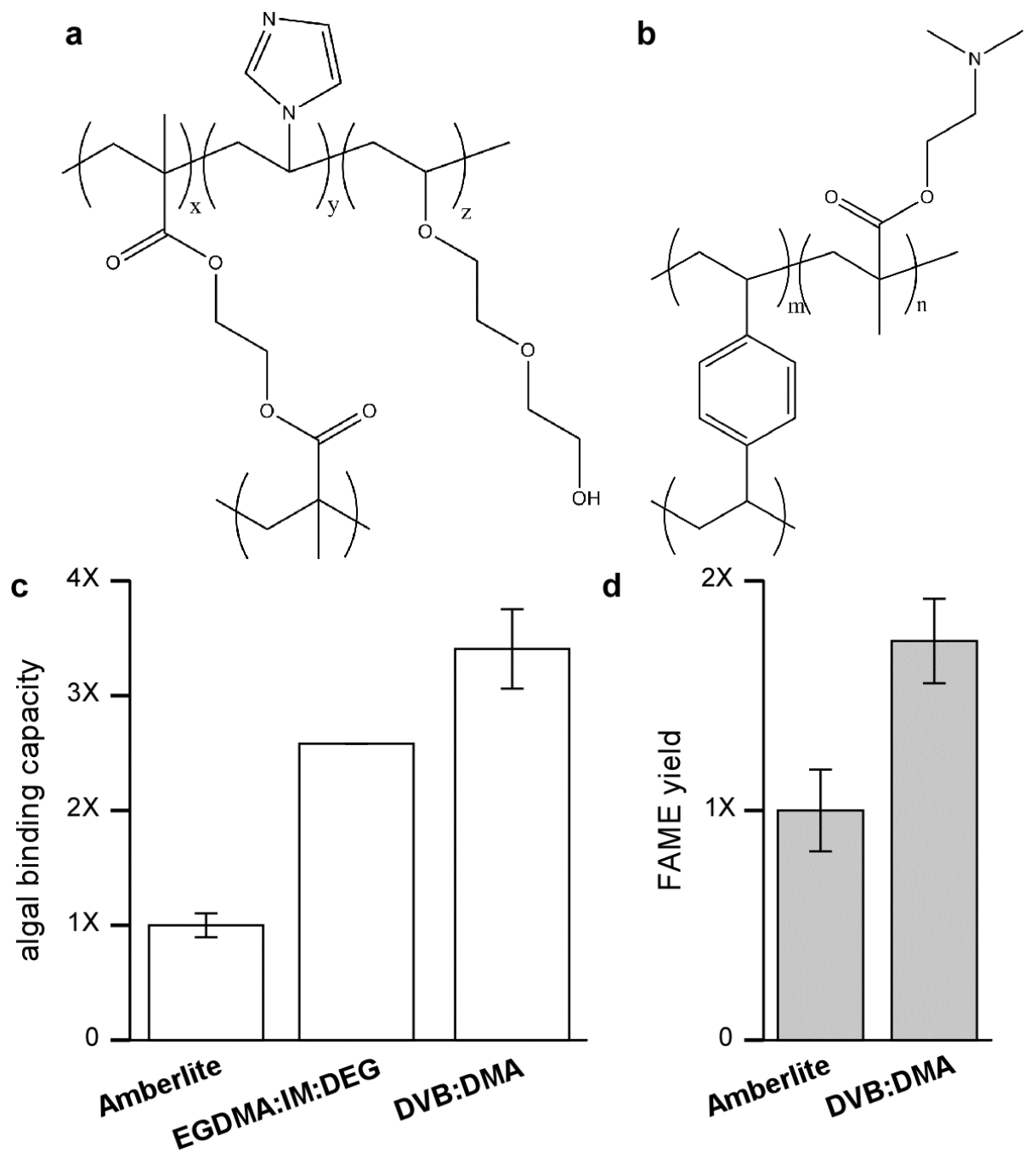

The results showed that the binding capacity for Neochloris was $37.0 \mathrm{mg} / \mathrm{g}$ resin whereas that for KAS 603 was $12.8 \mathrm{mg} / \mathrm{g}$ resin [Figure 1(b)]. In terms of efficiency of removal of algae from $1 \mathrm{~L}$ of $0.4 \mathrm{~g} / \mathrm{L}$ suspension, $10 \mathrm{~g}$ of Amberlite will sequester $93 \%$ of the Neochloris, whereas $30 \mathrm{~g}$ of Amberlite will sequester $96 \%$ of the KAS603 during a single pass through the column.

Although the difference in binding capacity could possibly be attributed to differences in surface charge density, studies to be detailed elsewhere show that the nature of the resin backbone, especially its hydrophobicity has a large impact on binding capacity [25]. In resins based on a methacrylate backbone, both Neochloris and KAS 603 showed similar binding capacities. Furthermore, binding capacity was greatly increased with the inclusion of glycol groups, which do not alter the charge characteristics of the resin but make it more hydrophilic. 
After binding, algae were eluted with $100 \mathrm{~mL}$ of $5 \%$ sulfuric acid in methanol. This treatment visibly removed algae from the resin and regenerated the resin. It appears that most of the algae was dissolved by the sulfuric acid/methanol reagent as shown by filtering an eluate of Neochloris through a pre-weighed PTFE filter (Millipore Omnipore, pore size $0.1 \mu \mathrm{m}$ ). Only $6.1 \%$ of the original DCW was recovered on the filter.

In order to determine how completely the resin was regenerated, resin columns were loaded with Neochloris [Figure 1(c) "fresh"] or KAS 603 [Figure 1(d) "fresh"] and eluted with fresh portions of sulfuric acid/methanol four times in succession. For comparison, parallel experiments were carried out where, after the initial binding and elution using fresh sulfuric acid/methanol solution, the reagent was then recycled for the three remaining cycles of algal binding and elution for both Neochloris [Figure 1(c) "used"] and KAS 603 [Figure 1(d) "used"]. With each cycle, the amount of algae bound to the resin was determined based on the $\mathrm{OD}_{680}$ as described above. Using fresh reagent, Neochloris after the initial binding and elution, binding capacity dropped to $97 \%$ of its initial value and remained constant thereafter. Using fresh reagent with KAS 603, binding dropped to $39 \%$ of its initial value after the first cycle but remained constant thereafter. When the experiments were carried out using the same sulfuric acid/methanol reagent for each cycle, the results were comparable to those obtained using the fresh reagent [Figures 1(c) and 1(d) "used").

The results show that the sulfuric acid/methanol reagent can be used for multiple cycles before it is consumed or rendered ineffective. Eventually methanol will be consumed while products such as glycerol, FAME, and other molecules will build up in the recycled sulfuric acid/methanol reagent. Unused methanol, being quite volatile would be easy to remove from the mixture. FAME, as we detail below, can also be easily removed. It will be interesting to see if the recycled reagent can be further fractionated and if potentially high value compounds might also be isolated from the mixture.

The drop in binding capacity of Amberlite for KAS 603 is not understood. It is not due to the action of the sulfuric acid/methanol reagent alone since pretreatment of the resin with sulfuric acid/methanol prior to initial binding of algae does not lower the binding capacity. A similar drop in binding capacity is not seen with the high capacity anion exchange resins based on a more polar methacrylate backbone [25]. Perhaps then the best explanation relates to the hydrophobicity of the divinylbenzene that interacts with hydrophobic components in the algae thereby either masking some of the charges on the resin or sterically interfering with binding.

\subsection{Conversion of Algal Lipids to FAME}

Since algae were eluted off the resin by $5 \%$ sulfuric acid in methanol, a reagent that catalyzes the transesterification of esterified fatty acid to FAMEs, tests were carried out to measure conversion of lipids in the eluate to FAME. To quantify FAME and other lipids, normal-phase HPLC was used in conjunction with an evaporative light scattering detector and mass spectrometry (HPLC-ELSD/MS; Supplementary Figure S3) [24]. The advantage here is that one can rapidly measure the amount of FAME generated, its fatty acid composition, and the amount of residual triacylglycerol starting material present in the reaction product as well as in crude total lipid extracts (Supplementary Figure S4). In the reaction product, the presence of residual TAG is an indicator that the transesterification reaction did not reach completion. Preliminary tests carried out by extracting the 
sulfuric acid methanol eluate with hexane to obtain the products at various times after elution showed that $12 \mathrm{~h}$ at room temperature was sufficient to consume all the TAG. What remains in the extract are mainly saturated hydrocarbons, which have been characterized in more detail elsewhere [24], and FAME (Supplementary Figure S4a).

As a reference, algal lipids were converted to FAME by a two step procedure that entailed treatment of dry algal pellets with base to hydrolyze fatty acid esters followed by re-esterification of free fatty acids in sulfuric acid and methanol [26]. The results showed $21.7 \%, 20.9 \%$, and $35.2 \%$ of dry weights were recovered as FAME for healthy Neochloris, stressed Neochloris, and KAS 603 respectively [Figure 2(a)]. Acid-catalyzed transesterification of resin-bound algae resulted in $13.6 \%, 6.9 \%$, and $37.6 \%$ of dry weight recovered as FAME for healthy Neochloris, stressed Neochloris, and KAS 603 respectively. For comparison, FAME synthesis yields are shown alongside total lipid extract amounts [Figure 2(a)]. Crude lipid extract constituted 31.4\% of total dry weight for healthy Neochloris, 35.4\% for stressed Neochloris, and $41.3 \%$ for KAS 603. HPLC analysis of the crude lipid extract showed that TAG constituted $0.4 \%, 8.6 \%$, and $11.7 \%$ of dry weight for healthy Neochloris, stressed Neochloris, and KAS 603, respectively.

For KAS 603 comparable yields of FAME were obtained using either method and the total FAME was close to the weight of total lipid. For Neochloris, both methods generated substantially less FAME than total lipid and the resin-bound algae yielded only $60 \%$ of the FAME generated from the dried pellet. Clearly, substantially more FAME can be generated by direct transesterification than can be accounted for by TAG alone. For healthy Neochloris, there was hardly any TAG present in the extracts yet $15 \%-20 \%$ of the DCW could be recovered as FAME. For KAS $603,10 \%$ of the DCW was present as TAG but nearly $40 \%$ of the DCW was recovered as FAME. These data suggest that much of the FAME is derived from polar lipids such as glycolipids and phospholipids.

One of the surprising results of this study is the finding that Neochloris accumulates high amounts of TAG when subjected to nitrogen deprivation [23], yet with only a modest increase in total lipid. While TAG increased nearly ten-fold, total lipid still constituted approximately $20 \%$ of dry weight, and FAME yield from dried biomass was comparable between healthy and stressed Neochloris. The most notable difference between FAME generated from healthy and stressed Neochloris was found in the fatty acid composition. This can be seen by analyzing positive mode APCI mass spectra of the various FAME reactions. Mass signatures were determined based on the fragmentation behavior of FAME standards (Supplementary Figure S5). Fatty acyl groups in FAME were identified and quantified by positive mode APCI-MS, as shown in Supplementary Figure S6 and Supplementary Table S1. Figures 2(b) and 2(c) indicate a trend towards a higher degree of fatty acid saturation with increased TAG content. For example, healthy Neochloris, having little TAG content, yielded more $\mathrm{C} 18: 3$ and $\mathrm{C} 21: 4$ species and less $\mathrm{C} 16: 0, \mathrm{C} 18: 0$, and $\mathrm{C} 20: 0$ than the other two algal groups. In contrast, stressed Neochloris and KAS 603, having a higher TAG content, yielded more C16:0, C18:0, and C20:0, and less C18:3 and C21:4 than healthy Neochloris. This shift in TAG fatty acid composition from unsaturated to saturated species with nitrogen starvation has been reported previously [21]. 


\subsection{Acid versus Base Elution and Transesterification}

Synthesis of FAMEs can be catalyzed by either base or acid. The base-catalyzed reaction is faster but can also generate soaps [4]. The sulfuric acid-catalyzed reaction, while slower, is also better at esterifying free fatty acids by dehydration [27]. Heat can speed up the acid-catalyzed reaction but there is a tradeoff between speed and the cost of heating. The previous results showed that $5 \%$ sulfuric acid in methanol could both elute algae from the resin and convert lipids to FAME. To test how well a base-catalyzed transesterification reagent would work, algae bound to Amberlite was eluted with either potassium hydroxide/methanol or sodium methoxide/methanol. We found that FAME yields from the sodium hydroxide/methanol reaction were undetectable and yields from the sodium methoxide/methanol reagent were $4 \%$ lower than using the sulfuric acid/methanol reagent. The reduced levels of FAME may be due to residual water, as shown by Griffiths [20], or to binding and depletion of the hydroxide or methoxide ions from solution by the anion exchange resin. For acid-catalyzed transesterification, we found that ethanol could be substituted for methanol with essentially identical results (Supplementary Figure S7). Because of its low cost and wide availability, methanol is often used for biodiesel synthesis [4]. However, ethanol cost may decrease in the future as advances in bioethanol productions lead to increases in supply.

\subsection{Recovery of FAME from Transesterification Reagent using Hydrophobic Resin}

The results from Figure 1 showed that the sulfuric acid/methanol transesterification reagent could be reused multiple times for eluting algae from resin. We next tested whether recycling the sulfuric $\mathrm{acid} / \mathrm{methanol}$ reagent led to a progressive decrease in FAME synthesis in comparison to fresh sulfuric acid/methanol [Figure 3(a)]. For the recycled sulfuric acid/methanol solution, each eluate was extracted with hexane and analyzed for FAME content before it was reused. The results showed that both fresh and recycled sulfuric acid/methanol solutions gave comparable amounts of FAME during four successive cycles of algal loading and elution.

Reuse of the sulfuric acid/methanol solution would minimize chemical costs, and removal of the FAME with each cycle should promote product formation. Hexane extraction of FAME is a relatively cumbersome and time consuming process leading us to explore a simple hydrophobic resin-based method to collect the FAME generated during each cycle. For comparison, the sulfuric acid/methanol eluate was either directly extracted with hexane or passed over a hydrophobic ethylene glycol dimethacylate:hexyl methacrylate (EGDMA:HMA) resin [Figure 3(b)] which was then eluted with hexane in a separate recovery step. The results show that FAME recovery from the resin $(11.0 \%$ of DCW) was comparable to that obtained by direct hexane extraction [11.8\% of DCW; Figure 3(c)]. While the hydrophobic column was effective at collecting FAME on the lab bench scale, we suspect that other methods, such as a hollow fiber membrane extractor [28] would be more useful commercially.

\subsection{Resins with Higher Algal Binding Capacity for Direct Transesterification}

While these studies were initiated and largely carried through using Amberlite, it became apparent through efforts to develop better resins that the binding capacity of Amberlite for algae is generally 
quite low [25]. It is particularly low for KAS603, which gave the highest yields of FAME relative to its dried weight. A comparison of Amberlite to other functionalized resins we have generated shows that EGDMA-IM-DEG [Figure 4(a)] and DVB-DMA [Figure 4(b)] resins showed equilibrium binding of 2.6 and 3.4 times more KAS603 than Amberlite, respectively [Figure 4(c)]. Currently we have obtained binding capacities up to $150 \mathrm{mg}$ algae per $\mathrm{g}$ resin. While these resins can be eluted cleanly with the sulfuric acid/methanol reagent so as to generate FAME [Figure 4(d)], they were not designed for this purpose and are potentially susceptible to attack by the strong acid. Future resin designs will need to address the need for chemical stability under harsh conditions.

\subsection{Evaluation}

While we are not equipped to do a formal life cycle analysis and feel it would be premature given the improvements needed in resin technology, nevertheless a comparison of the proposed resin-based processing to current approaches seems in order. Figure 5 presents a general schematic for current algal processing schemes derived on published evaluations [5,29]. For the most part, the cost of growing algae would be the same. The use of resins could impact growth in terms of the need for nitrogen starvation or growth of monocultures as discussed below. Primarily, our one-step harvest to biodiesel approach would impact the most expensive steps in processing algae to biofuel, namely the harvesting and extraction steps.

Currently harvesting typically requires both pumping the algal suspension and some treatment such as centrifugation, flocculation or dissolved air flotation. Centrifugation and dissolved air flotation both have relatively large capital costs and substantial electricity. Where resins could make a difference is by eliminating the need for pumping water. While we have used resin beads for comparisons, we do not feel this is the best way to deploy them for harvesting. Resin beads are porous with a large internal volume that can trap water. The pores are typically $10-30 \mathrm{~nm}$ in diameter whereas the algae are on the order of $2-3 \mu \mathrm{m}$ in diameter so they cannot enter the resin. Our measurements show that after the bulk water is removed, about $100 \mathrm{mg}$ of water/gram resin is retained either inside the resin or entrained between the beads. A better alternative is to use thin films of resin where the internal volume is low compared to the surface area. We have proposed elsewhere a belt harvester type approach where a belt moves from the pond, where it picks up the algae to a vat which, in this case would contain sulfuric $\mathrm{acid} / \mathrm{methanol} \mathrm{[25].} \mathrm{In} \mathrm{that} \mathrm{study} \mathrm{we} \mathrm{estimated} \mathrm{that} \mathrm{a} \mathrm{bristled} \mathrm{belt} 1 \mathrm{~m}$ wide $\times 7.5 \mathrm{~m}$ long could collect $3 \mathrm{~kg}$ algae per one complete turn of the belt. Other modes are possible such as mesh bags containing nonporous resin-coated particles that float or are semisubmerged.

Once the algae is harvested and eluted, the conversion to biodiesel is direct. There is no need for lysing, further drying and solvent extraction, steps that are considered quite expensive [5]. Hexane extraction requires recovery of the hexane and a certain amount will be lost to the environment. Furthermore, hexane only recovers the neutral lipids (mainly TAG). Our experience at measuring TAG in pond-grown algae is that TAG levels rarely surpass $5 \% \mathrm{DCW}$ and we have never seen them greater than $12 \% \mathrm{DCW}$. One of the advantages of this approach is that FAME is generated from both polar and neutral lipids thereby increasing the yield from the algae. Our data suggest improvements of up to 4-fold were possible with an alga (KAS 603) that made 10\% TAG. 
Figure 5. Sequence of steps to obtain fuel from algae. (a) A conventional sequence of steps is given for the obtaining biodiesel from algae. (b) A sequence of processing steps using a resin-based approach. Both sequences are essentially the same in terms of growth. Conventional harvesting involves pumping of algal suspension and some other treatment such as centrifugation, dissolved air flotation or flocculation with chemicals. For the resin-based approach the resin will collect algae leaving most of the water behind. One possible mechanism is to use a belt harvester employing a resin- coated belt. For the conventional approach a possible lysis step between harvest and extraction has been included since it can improve extraction efficiency. For solvent extraction it is usually necessary to dry the algae. In the resin-based approach drying and conventional solvent extraction is eliminated. In this scenario, biodiesel would be produced on site so the cost of transportation of the extracted oil to a biodiesel plant would also be eliminated. Since biodiesel is generated with or without employing resins, the cost of that step would be similar in either scenario.

\section{A Conventional}

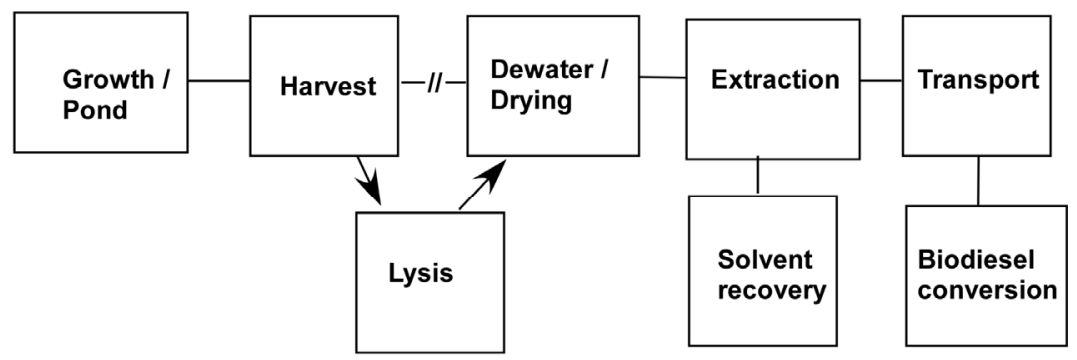

B Resin-based

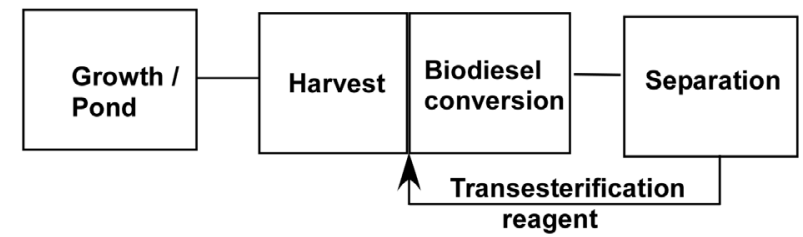

The improved yield could affect several aspects of the algal growth process. Firstly, our data suggested that there was not so much to be gained by nitrogen starvation. Both starved and unstarved algae contained similar amounts of total lipid. Admittedly, the spectrum of fatty acids was different and this might be a factor. Secondly, resin-based conversion of total lipid to biodiesel might make it less important for growers to maintain a monoculture of high TAG-producing algae. Of course, high TAG production is a benefit but it is difficult to maintain monocultures in open ponds or in potentially other attractive scenarios where algae are grown in conjunction with wastewater treatment plants.

Finally production of biodiesel onsite eliminates the transportation cost of moving algal oil to a biodiesel plant. While the biodiesel must be recovered from the sulfuric acid methanol, we believe this can be done using a low solvent or even solventless hollow fiber extraction system. In commercial biodiesel plants, the glycerol separates from the biodiesel layer and the separation is facilitated by water. This may be possible as well but more study is needed to determine how best to fractionate the spent sulfuric acid/ methanol mixture. There will be many cellular components in the final sulfuric acid/methanol solution, some of which may be valuable as side products. 


\subsection{Future Directions}

Despite the potential advantages, more work is needed to optimize the resin approach. The use of resin beads is convenient for comparing properties such as binding capacity and fouling for both commercial and our own laboratory-synthesized resins. Here we used a commercially available strong cation exchange resin where sulfuric acid/methanol is used to elute the resin. It is entirely feasible to use a weakly basic anion exchange resin where raising the $\mathrm{pH}$ releases the algae from the resin [25]. At lower $\mathrm{pH}(7-8)$ these resins are positively charged and effectively bind algae. Raising the $\mathrm{pH}$ to $\sim \mathrm{pH} 10$ or above deprotonates the resin rendering it uncharged such that algae are released. Sodium methoxide in methanol, a strongly basic commercial transesterification reagent, would raise the $\mathrm{pH}$, thereby releasing the algae and catalyzing the transesterification reaction. Here also, it would be critical to use a nonporous resin that did not entrain water.

Future directions are aimed at determining which approach, using strong or weak anion exchange resins is more effective in terms of binding capacity, fouling, and FAME yields. It will also be important to develop new modes for use of the resins such as thin film coatings or resin coated particles that do not entrain water. Finally, more studies of the transesterification reaction are in order since, as shown with Neochloris, the one step conversion to FAME was not quite complete and was further affected by the nitrogen starvation.

\section{Experimental Section}

Chloroform, ethanol, ethyl acetate, hexane, isopropanol, isooctane, methanol, and 2-ethoxyethanol (2-EE; Acros, Bridgewater, NJ, USA) were obtained from Fisher (Waltham, MA, USA) and were either HPLC or ACS reagent grade. Sulfuric acid and potassium hydroxide were reagent grade (Fisher). Sodium methoxide in methanol (0.5M), methyl oleate, methyl palmitate, di(ethylene glycol) vinyl ether (DEG), 2-dimethylamino methacrylate (DMA), divinylbenzene (DVB), ethylene glycol dimethacrylate (EGDMA), hexyl methacrylate (HMA), vinyl imidazole (IM), and azobisisobutyronitrile (AIBN) were obtained from Sigma-Aldrich (St. Louis, MO, USA). Triolein was obtained from Nuchek Prep (Elysian, MN, USA) and mineral oil (Squibb) was purchased locally.

All HPLC solvents were degassed and filtered through 0.5 micron PTFE filters (Ominpore, Waters Corp, Milford, MA, USA) prior to use. Isooctane was dried by storing over calcium hydride and filtered before use. Amberlite ${ }^{\circledR}$ ion exchange resin CG-400 (100-200 mesh, chromatographic grade; Mallinckrodt, St. Louis, MO, USA) was prepared by washing and settling in distilled water in order to remove fines, then dried at $55^{\circ} \mathrm{C}$.

\subsection{Algal Cultivation and Harvest}

Neochloris oleoabundans (UTEX LB 1185) was obtained from the University of Texas at Austin Algal Culture Collection and KAS 603, a saltwater species of Chlorella, was provided by Kuehnle Agro Systems. Neochloris was cultivated in freshwater Bold 3N (B3N) medium [30] using an airlift bioreactor illuminated with cool white fluorescent lights on a 12:12 light:dark cycle and aerated with ambient air using an oil-free diaphragm pump. KAS 603 was cultivated in the same way using f/2 saltwater medium [31]. For nitrogen starvation experiments, Neochloris was grown for 14 days in B3N 
media, harvested by centrifugation, resuspended in sodium nitrate-free $\mathrm{B} 3 \mathrm{~N}$, and cultured for an additional 21 days. For resin binding and FAME synthesis, algae were harvested by centrifugation and resuspended in distilled water to concentration of $0.4 \mathrm{~g} / \mathrm{L}$.

\subsection{Algal Mass Quantification}

Routine determination of algal dry cell weight (DCW) was obtained by measuring the optical density of chlorophyll at $680 \mathrm{~nm}\left(\mathrm{OD}_{680}\right)$ using a spectrophotometer (Shimadzu UV-265). The conversion of $\mathrm{OD}_{680}$ to $\mathrm{DCW}$ was accomplished by generating a dilution series for each species, recording the $\mathrm{OD}_{680}$, and then collecting samples onto pre-weighed cellulose acetate membranes (Pall Co., Port Washington, NY, USA), which were then dried in a vacuum oven $\left(15 \mathrm{in} . \mathrm{Hg} ., 60^{\circ} \mathrm{C}\right)$ for $12 \mathrm{~h}$ before obtaining the final weights. To avoid optical filtering effects, samples were diluted, if need be, such that the $\mathrm{OD}_{680}$ was always less than 1.8.

\subsection{Algal Binding and FAME Generation}

For algae binding and FAME conversion studies, $10 \mathrm{~mL}$ polypropylene columns were loaded with $2 \mathrm{~g}$ of Amberlite resin and then washed with $1 \mathrm{M}$ hydrochloric acid followed by distilled water. To load the resin, $200 \mathrm{~mL}$ algal suspension containing $80 \mathrm{mg}$ algae (a slight excess; based on the $\mathrm{OD}_{680}$ of the algae suspension) was passed through the resin. The $\mathrm{OD}_{680}$ of the flow through was then measured to determine the DCW of algae bound to the resin.

For transesterification and elution of algae from the resin, excess water was removed from column by vacuum, followed by the addition of $100 \mathrm{~mL}$ of transesterification reagent. For elution of algae and transesterification, reagents tested included $5 \% \mathrm{w} / \mathrm{v}$ sulfuric acid in either methanol or ethanol, $5 \% \mathrm{w} / \mathrm{v}$ potassium hydroxide in methanol, or $0.1 \mathrm{M}$ sodium methoxide in methanol. The eluate was collected and the transesterification reaction continued at room temperature for $12 \mathrm{~h}$. FAME was extracted sequentially with two $20 \mathrm{~mL}$ portions of hexane. The combined hexane extracts were then dried by rotary evaporation and resuspended in $1 \mathrm{~mL}$ hexane:isopropanol $(3: 1, \mathrm{v} / \mathrm{v})$ for HPLC analysis.

For comparison, FAME was generated directly from dried algal biomass, based on the method described by O'Fallon et al. [26]. Algal pellets containing approximately $30 \mathrm{mg}$ algae in preweighed tubes were dried using a SpeedVac concentrator (Savant) for $12 \mathrm{~h}$ at $10^{-1}$ Torr with heat. Final tube weight was then measured to obtain an accurate algal DCW. The dried algal pellet was suspended in methanol and transferred to a glass centrifuge tube where methanol was added to a final volume of $5.3 \mathrm{~mL}$. Lipids were then saponified by adding $0.7 \mathrm{~mL}$ of $10 \mathrm{~N}$ potassium hydroxide and heating at $55^{\circ} \mathrm{C}$ for $1.5 \mathrm{~h}$. Once the samples cooled, lipids were re-esterified by adding $0.6 \mathrm{~mL}$ of concentrated sulfuric acid to the same methanol suspension and heating again at $55{ }^{\circ} \mathrm{C}$ for $1.5 \mathrm{~h}$. After cooling, FAME was extracted with $2 \mathrm{~mL}$ of hexane using a centrifuge to force the separation of the mixture into two layers. The upper hexane layer was then transferred to a vial for HPLC analysis.

\subsection{Total Lipid Extraction from Algae}

Total lipid was determined as described by Jones et al. [24] in order to assess the oil available for biodiesel synthesis for both algal species. Briefly, dried algal pellets (approximately $30 \mathrm{mg}$ ) were 
extracted with $20 \mathrm{~mL}$ of 2 -EE for $30 \mathrm{~min}$ at $60{ }^{\circ} \mathrm{C}$ with continuous stirring. The solution was then filtered through $0.47 \mu \mathrm{m}$ PTFE membrane (Millipore) and the residual biomass was extracted with a second $20 \mathrm{~mL}$ portion of 2-EE. After filtration, the two filtrates were combined, dried under vacuum, and weighed.

\subsection{Hydrophobic Resin Synthesis and FAME Recovery}

Hydrophobic resins used in this study were synthesized in bulk by combining $8 \mathrm{~g}$ of EGDMA and $2 \mathrm{~g}$ of HMA with $10 \mathrm{~mL}$ of toluene as the porogen. Resin synthesis was carried out in a round bottom flask fitted with an argon bubbler and heated to $60{ }^{\circ} \mathrm{C}$ with constant stirring. Polymerization was initiated by addition of $1 \mathrm{~mol}$ percent AIBN and continued until the mixture formed a brittle solid. The polymer was then dried at $55^{\circ} \mathrm{C}$ for $12 \mathrm{~h}$, scraped from the flask and ground by mortar and pestle. The crushed resin was then sized between \#35 and \#170 meshes to obtain beads of approximately $100-500 \mu \mathrm{m}$ diameter.

As an alternative to solvent extraction, FAME generated by sulfuric acid/methanol transesterification was collected by passing the reaction mixture over a hydrophobic resin bed. Here, the $100 \mathrm{~mL}$ of sulfuric acid/methanol used to elute algae off the Amberlite resin was passed through a $10 \mathrm{~mL}$ polypropylene column containing $2 \mathrm{~g}$ of EDGMA-HMA resin. The resin was then eluted with $50 \mathrm{~mL}$ of hexane-ethyl acetate $(3: 1, \mathrm{v} / \mathrm{v})$ solvent that was subsequently removed by rotary evaporation and the residue resuspended in $1 \mathrm{~mL}$ hexane-isopropanol (3:1, v/v) for HPLC analysis. For comparison, parallel samples of algae bound to Amberlite were eluted with $100 \mathrm{~mL}$ sulfuric acid/methanol and extracted with two $20 \mathrm{~mL}$ portions of hexane. After mixing and phase separation, the upper organic phase was recovered, dried by rotary evaporation, and the residue resuspended in $1 \mathrm{~mL}$ hexane-isopropanol $(3: 1, \mathrm{v} / \mathrm{v})$ for HPLC analysis.

\subsection{Functionalized Resin Synthesis and Comparison with Amberlite}

Functionalized weak anion exchange resins used in this study were synthesized in the manner stated above, using $6 \mathrm{~g}$ of EGDMA, $3 \mathrm{~g}$ of IM, and $1 \mathrm{~g}$ of DEG (EGDMA:IM:DEG), or $6 \mathrm{~g}$ of DVB and $4 \mathrm{~g}$ of DMA (DVB:DMA), with a solution of $5 \mathrm{~mL}$ of toluene (porogen) and $5 \mathrm{~mL} \mathrm{3 \%}$ acetic acid (dispersing agent).

Steady-state binding capacity was determined by agitating $2 \mathrm{~g}$ of resin in a $500 \mathrm{~mL}$ flask containing $100 \mathrm{~mL}$ of KAS603 at $0.4 \mathrm{~g} / \mathrm{L}$ concentration for $15 \mathrm{~min}$. The suspension containing unbound algae was filtered off and resin transferred into columns. FAME was directly transesterified from the resin as stated previously using $100 \mathrm{~mL}$ of $5 \%$ sulfuric acid-methanol. For comparison, algae was bound and eluted from a parallel set of Amberlite resin. Because of the higher binding capacity of resin, columns were eluted for 3 consecutive cycles using the same transesterification reagent to ensure complete removal. After reaction for $12 \mathrm{~h}$ as room temperature, FAME was recovered by extracting twice with $20 \mathrm{~mL}$ of hexane. The recovered organic phase was dried by rotary evaporation, and the residue resuspended in $1 \mathrm{~mL}$ hexane:isopropanol $(3: 1, \mathrm{v} / \mathrm{v})$ for HPLC analysis. 


\subsection{Quantification and Characterization by HPLC-ELSD/MS}

Lipid composition and FAME content were analyzed as previously described [24], using an HPLC (Surveyor LC Pump and Autosampler Plus, Thermo Finnegan, USA) coupled to both ELSD (Sedere Sedex 75) and quadrupole mass spectrometer (Thermo Finnigan MSQ) using a 10:1 line splitter (Analytical Instruments, USA). Xcalibur software controlled operation of the autosampler, pump, and mass spectrometer. ELSD analog data was acquired through an A/D data acquisition box (Agilent Technologies, SS420X) and RS232 PCI data acquisition card (Sea Level Systems, 7406S).

Lipid standards and extracts were resolved using a YMC Pack PVA-Sil-NP column (250 $\mathrm{mm} \times 4.6 \mathrm{~mm}$ I.D., $5 \mu \mathrm{m}$ bead size) protected by a Waters Guard Pak ${ }^{\mathrm{TM}}$ guard column containing Nova-Pak ${ }^{\mathrm{TM}}$ silica inserts. The solvent program is given in Table 1 . ELSD was run at $30{ }^{\circ} \mathrm{C}$ at gain setting 8. Mass spectrometer was run in APCI positive mode with probe temperature of $400{ }^{\circ} \mathrm{C}$.

Table 1. Resolution of algal lipid classes using normal-phase HPLC. Normal-phase HPLC mobile phase gradient method using a three-solvent system of iso-octane (A), ethyl acetate (B), and isopropanol:methanol:water $(3: 3: 1, \mathrm{v} / \mathrm{v} / \mathrm{v})+0.1 \%$ acetic acid (C).

\begin{tabular}{ccccc}
\hline time (min) & flow rate (mL/min) & A (\%) & B (\%) & C (\%) \\
\hline 0 & 1.5 & 100 & 0 & 0 \\
5 & 1.5 & 98 & 2 & 0 \\
15 & 1.5 & 75 & 25 & 0 \\
19 & 1.5 & 20 & 80 & 0 \\
24 & 1.5 & 0 & 100 & 0 \\
32 & 1.3 & 0 & 50 & 50 \\
38 & 1.0 & 0 & 15 & 85 \\
43 & 1.0 & 0 & 0 & 100 \\
52 & 1.0 & 0 & 100 & 0 \\
54 & 1.0 & 0 & 100 & 0 \\
60 & 1.5 & 90 & 10 & 0 \\
64 & 1.5 & 100 & 0 & 0 \\
74 & 1.5 & 100 & 0 & 0 \\
\hline
\end{tabular}

\section{Conclusions}

Anion exchange resins can be used as a simple and inexpensive support for one-step algal harvest and biodiesel generation. The yields of FAME are greatly improved over methods that first isolate the TAG fraction since polar lipids also contribute to the FAME pool. Both the resin and the transesterification reagent can be reused for numerous cycles with the resultant FAME collected during each cycle. Although the basic principles have been demonstrated there is much room for improvement, especially in the design of resins. Although resins bind to algae based on surface charge, there are clearly additional issues that impact binding capacity, fouling and FAME conversion that are specific to different species of algae.

\section{Acknowledgments}

This work was supported in part by OpenAlgae. 


\section{References}

1. Scott, S.A.; Davey, M.P.; Dennis, J.S.; Horst, I.; Howe, C.J.; Lea-Smith, D.J.; Smith, A.G. Biodiesel from algae: Challenges and prospects. Curr. Opin. Biotechnol. 2010, 21, 277-286.

2. Durrett, T.P.; Benning, C.; Ohlrogge, J. Plant triacylglycerols as feedstocks for the production of biofuels. Plant J. 2008, 54, 593-607.

3. Hossain, A.B.M.S.; Salleh, A.; Boyce, A.N.; Chowdhury, P.; Naqiuddin, M. Biodiesel fuel production from algae as renewable energy. Am. J. Biochem. Biotechnol. 2008, 4, 250-254.

4. Gerpen, J.V. Biodiesel processing and production. Fuel Process Technol. 2005, 86, 1097-1107.

5. Lardon, L.; Hélias, A.; Sialve, B.; Steyer, J.-P.; Bernard, O. Life-Cycle Assessment of Biodiesel Production from Microalgae. Environ. Sci. Technol. 2009, 43, 6475-6481.

6. Li, Y.; Horsman, M.; Wu, N.; Lan, C.Q.; Dubois-Calero, N. Biofuels from microalgae. Biotechnol. Prog. 2008, 24, 815-820.

7. Olaizola, M. Commercial development of microalgal biotechnology: From the test tube to the marketplace. Biomol. Eng. 2003, 20, 459-466.

8. Salim, S.; Bosma, R.; Vermue, M.H.; Wijffels, R.H. Harvesting of microalgae by bio-flocculation. J. Appl. Phycol. 2010, 1-7.

9. Schurchardt, U.; Sercheli, R.; Vargas, R.M. Transesterification of vegetable oils: A review. J. Braz. Chem. Soc. 1998, 9, 199-210.

10. Bikou, E.; Louloudi, A.; Papayannakos, N. The effect of water on the transesterification kinetics of cotton seed oil with ethanol. Chem. Eng. Technol. 1999, 22, 70-75.

11. Johnson, M.B.; Wen, Z. Production of biodiesel fuel from the microalga Schizochytrium limacinum by direction transesterification of algal biomass. Energy Fuels 2009, 23, 5179-5183.

12. Komers, K.; Machek, J.; Stioukal, R. Biodiesel from rapeseed oil and KOH, 2. Composition of solution of $\mathrm{KOH}$ in methanol as reaction partner of oil. Eur. J. Lipid Sci. Technol. 2001, 103, 359-362.

13. Kusdiana, D.; Saka, S. Effects of water on biodiesel fuel production by supercritical methanol treatment. Bioresour. Technol. 2004, 91, 289-295.

14. Ma, F.; Clements, L.D.; Hanna, M.A. The effects of catalyst, free fatty acids, and water on transesterification of beef tallow. Trans. ASAE 1998, 41, 1261-1264.

15. Clarens, A.F.; Resureccion, E.P.; White, M.A.; Colosi, L.M. Environmental life cycle comparison of algae to other bioenergy feedstocks. Environ. Sci. Technol. 2009, 44, 1813-1819.

16. Armenta, R.E.; Scott, S.D.; Burja, A.M.; Radianingtyas, H.; Barrow, C.J. Optimization of fatty acid determination in selected fish and microalgal oils. Chromatographia 2009, 70, 629-636.

17. Haas, M.J.; Wagner, K. SImplifying biodiesel production: The direct or in situ transesterification of algal biomass. Eur. J. Lipid Sci. Technol. 2011, 113, 1219-1229.

18. Johnson, E.A.; Liu, Z.; Salmon, E.; Hatcher, P.G. One-step Conversion of Algal Biomass to Biodeisel with Formation of an Algal Char as Potential Fertilizer. In Advanced Biofuels and Bioproducts; Lee, J.W., Ed.; Springer: New York, NY, USA, 2012.

19. Koberg, M.; Cohen, M.; Ben-Amotz, A.; Gedanken, A. Bio-diesel production directly from the microalgae biomass of Nannochloropsis by microwave and ultrasound radiation. Bioresour. Technol. 2011, 102, 4265-4269. 
20. Griffiths, M.J.; Hille, R.P.; Harrison, S.T.L. Selection of direct transesterification as the preferred method for assay of fatty acid content of microalgae. Lipids 2010, 45, 2010.

21. Braithwaite, A.; Smith, F.J. Chromatographic Methods; Springer: London, UK, 1996.

22. Onyancha, D.; Mavura, W.; Ngila, J.C.; Ongoma, P.; Chacha, J. Studies of chromium removal from tannery wastewaters by algae biosorbents, Spirogyra condensata and Rhizoclonium hieroglyphicum. J. Haz. Mat. 2008, 158, 605-614.

23. Tornabene, T.G.; Holzer, G.; Lien, S.; Burris, N. Lipid composition of the nitrogen starved green alga Neochloris oleoabundans. Enz. Microb. Technol. 1983, 5, 435-440.

24. Jones, J.; Manning, S.; Montoya, M.; Keller, K.; Poenie, M. Extraction of algal lipids and their analysis by HPLC and mass spectrometry. J. Am. Oil Chem. Soc. 2012, 89, doi:10.1007/s11746012-2044-8.

25. Jones, J.; Poenie, M. Resins that reversibly bind algae for harvesting and concentration. Environ. Prog. 2012, submitted for publication.

26. O'Fallon, J.V.; Busboom, J.R.; Nelson, M.L.; Gaskins, C.T. A direct method for fatty acid methyl ester synthesis: Application to wet meat tissues, oils, and feestuffs. J. Anim. Sci. 2007, 85, 1511-1521.

27. Chisti, Y. Biodiesel from microalgae. Biotechnol. Adv. 2007, 25, 294-306.

28. Seibert, F.; Poenie, M. Non-Dispersive Process for Insoluble Oil Recovery From Aqueous Slurries. U.S. Patent 2011/0174734 A1, 21 July 2011.

29. Sander, K.; Murthy, G.S. Life cycle analysis of algae biodiesel. Int. J. Life Cycle Assess. 2010, 15, 704-714.

30. Brown, M.R.; Bold, H.C. Comparative Studies of the Algal Genera Tetracystis and Chlorococcum; University of Texas Publication: Austin, UT, USA, 1964; p. 213.

31. Jeffrey, S.W.; LeRoi, J.M. Simple Procedures for Growing SCOR Reference Microalgal Cultures; UNESCO: Marseille, France, 1997.

(C) 2012 by the authors; licensee MDPI, Basel, Switzerland. This article is an open access article distributed under the terms and conditions of the Creative Commons Attribution license (http://creativecommons.org/licenses/by/3.0/). 
Copyright of Energies (19961073) is the property of MDPI Publishing and its content may not be copied or emailed to multiple sites or posted to a listserv without the copyright holder's express written permission. However, users may print, download, or email articles for individual use. 